\title{
Preoperative functional MRI use in neurooncology patients: a clinician survey
}

\author{
Brittany M. Stopa, MPH, ${ }^{1}$ Joeky T. Senders, MD,1,2 Marike L. D. Broekman, MD, PhD, JD, ${ }^{3}$ \\ Mark Vangel, PhD, ${ }^{4}$ and Alexandra J. Golby, MD ${ }^{4,5}$
}

${ }^{1}$ Computational Neuroscience Outcomes Center, Department of Neurosurgery, Brigham and Women's Hospital, Harvard Medical School, Boston, Massachusetts; '2Department of Neurosurgery, University Medical Center, Utrecht, The Netherlands; ${ }^{3}$ Department of Neurosurgery, Haaglanden Medical Center, The Hague, The Netherlands; and Departments of ${ }^{4}$ Radiology and ${ }^{5}$ Neurosurgery, Brigham and Women's Hospital, Harvard Medical School, Boston, Massachusetts

OBJECTIVE Functional MRI (fMRI) is increasingly being investigated for use in neurosurgical patient care. In the current study, the authors characterize the clinical use of fMRI by surveying neurosurgeons' use of and attitudes toward fMRI as a surgical planning tool in neurooncology patients.

METHODS A survey was developed to inquire about clinicians' use of and experiences with preoperative fMRI in the neurooncology patient population, including example case images. The survey was distributed to all neurosurgical departments with a residency program in the US.

RESULTS After excluding incomplete surveys and responders that do not use fMRI $(n=11), 50$ complete responses were included in the final analysis. Responders were predominantly from academic programs (88\%), with 20 years or more in practice $(40 \%)$, with a main area of practice in neurooncology $(48 \%)$ and treating an adult population $(90 \%)$. All 50 responders currently use fMRI in neurooncology patients, mostly for low- (94\%) and high-grade glioma (82\%). The leading decision factors for ordering fMRI were location of mass in dominant hemisphere, location in a functional area, motor symptoms, and aphasia. Across 10 cases, language fMRI yielded the highest interrater reliability agreement (Fleiss' kappa 0.437). The most common reasons for ordering fMRI were to identify language laterality, plan extent of resection, and discuss neurological risks with patients. Clinicians reported that fMRI results were not obtained when ordered a median $10 \%$ of the time and were suboptimal a median $27 \%$ of the time. Of responders, $70 \%$ reported that they had ever resected an fMRI-positive functional site, of whom 77\% did so because the site was "cleared" by cortical stimulation. Responders reported disagreement between $\mathrm{fMRI}$ and awake surgery $30 \%$ of the time. Overall, $98 \%$ of responders reported that if results of $\mathrm{fMRI}$ and intraoperative mapping disagreed, they would rely on intraoperative mapping.

CONCLUSIONS Although $\mathrm{fMRI}$ is increasingly being adopted as a practical preoperative planning tool for brain tumor resection, there remains a substantial degree of discrepancy with regard to its current use and presumed utility. There is a need for further research to evaluate the use of preoperative fMRI in neurooncology patients. As fMRI continues to gain prominence, it will be important for clinicians to collectively share best practices and develop guidelines for the use of $\mathrm{fMRI}$ in the preoperative planning phase of brain tumor patients.

https://thejns.org/doi/abs/10.3171/2019.11.FOCUS19779

KEYWORDS brain mapping; functional MRI; neurooncology; neurosurgery; clinician survey

$\mathrm{F}$ UNCTIONAL MRI (fMRI) has rapidly gained prominence since the first successful fMRI scan was performed in 1991. ${ }^{1}$ By imaging the ratio of oxygenated and deoxygenated hemoglobin, T2*-weighted MR images are able to measure changes in cerebral blood flow, a surrogate for neuronal activation. fMRI has enabled visualization of brain activation and expanded our understanding of human brain function in both healthy and diseased states. ${ }^{9}$ Since its inception as a medical imaging modality, fMRI has attracted significant attention for its potential clinical applications, especially for presurgical mapping. ${ }^{12}$

A large volume of academic research has reported the potential utility of fMRI, including more than 20,000 publications in the first 20 years of its existence. ${ }^{12}$ The current body of research has done much to improve imaging hardware, methodology, processing methods, image display

ABBREVIATIONS DTI = diffusion tensor imaging; $f M R I=$ functional MRI; IQR = interquartile range.

SUBMITTED September 30, 2019. ACCEPTED November 5, 2019.

INCLUDE WHEN CITING DOI: 10.3171/2019.11.FOCUS19779. 
software, and applications of fMRI., ${ }^{1,5}$ These technological advances have contributed to fMRI becoming a useful clinical functional brain-mapping tool, especially for brain tumor patients. ${ }^{6,11,13}$ However, the question remains whether clinical adoption of fMRI has matched this potential. ${ }^{12,14}$ While the clinical adoption of fMRI for epilepsy patients has been examined, ${ }^{3}$ for neurooncology patients it has not. We therefore set out in the present study to investigate neurosurgeons' clinical adoption of fMRI for preoperative planning in neurooncology patients.

\section{Methods \\ Study Design}

This study was designed as a clinician survey. It was approved and overseen by the Partners IRB (Brigham and Women's Hospital). All clinician participants provided informed consent (see question 1, Supplement 1).

\section{Survey}

The survey (Supplement 1) consisted of several sections, including neurosurgeon demographics, patient population, decision factors for ordering fMRI, case studies, intended purpose of ordering fMRI, feasibility of obtaining fMRI results, use of fMRI results, and comparison to other intraoperative brain mapping techniques. A previous survey of fMRI use in epilepsy patients was referenced in this survey design. ${ }^{3}$

The survey did not use responder identifiers and was made available on Qualtrics (www.qualtrics.com). Questions were organized with logical hierarchy, allowing answers from some key questions to trigger additional questions to appear. The question formats included multiple choice, some with single answer and some with multiple answer, and sliding scale. All questions were optional, and responders could proceed through the survey if they chose to skip questions. The MR images used in the case studies in the survey were de-identified, and their use was approved by the IRB.

\section{Data Collection}

The survey was open between September 1, 2018, and February 28, 2019. We emailed invitations to all neurosurgical residency program directors, residency program administrators, and department chiefs in the US. Using a snowball sampling approach, ${ }^{7}$ we requested that these individuals share the survey link with the faculty, fellows, and residents in their departments.

\section{Data Analysis}

Results of the survey were downloaded from Qualtrics and manually cleaned before being processed in RStudio (version 1.1.463, RStudio, Inc.). Survey question responses are summarized in frequencies and percentages, or median and interquartile range (IQR), as appropriate. Case studies were used to examine the agreement between neurosurgeons in ordering fMRI testing procedures. The agreement was quantified by means of the Fleiss' kappa statistic. ${ }^{10}$ This statistic measures the strength of the interrater reliability between -1 and 1 , taking into account the possibility of agreement due to chance. Based on this statistic, the strength of agreement could be classified into less than chance $(<0)$, slight $(0.01-0.20)$, fair $(0.21-0.40)$, moderate $(0.41-0.60)$, substantial $(0.61-0.80)$, and near perfect (0.81-1.00) based on interpretation guidelines set out in the study by Viera and Garrett..$^{15}$ The interrater reliability was calculated for the case studies overall, as well as the agreement within specific functional domains and individual cases.

\section{Results}

\section{Excluded Responders}

A total of 75 survey responses were started, but only 50 responses were completed and used in the final analysis. Of the responders excluded, there were 11 who reported not using fMRI, 7 who did not complete the survey (average progress $40 \%$ ), 6 who left the complete survey empty, and 1 who did not consent to the survey (Supplement 1, question 1; Fig. 1).

Of those responders who do not use fMRI, their reasons include the following: equipment not available $(\mathrm{n}=$ 3 ), too difficult to perform ( $(n=2)$, do not think it can map language function $(\mathrm{n}=2)$, no buy-in from radiology team $(n=2)$, not sensitive enough $(n=1)$, not specific enough $(\mathrm{n}=1)$, not a reliable method $(\mathrm{n}=1)$, not needed in my specialty $(n=1)$, usually not needed $(n=1)$, and not the decision-maker $(n=1 ;$ Fig. 1$)$. Responders were permitted to choose more than one response.

\section{Responder Demographics}

Survey responders were predominantly from academic programs $(88 \%)$, male $(86 \%)$, with 20 or more years in practice $(40 \%)$, and with a primary practice in an adult population (90\%). Of those who work with a pediatric population, the median youngest age they thought it was possible to use fMRI was 7 years (IQR 6-8 years). All responders have neurooncology as an area of practice, and $48 \%$ reported that neurooncology is their main area of practice (Table 1).

\section{Current Use of fMRI}

All included responders currently use fMRI in neurooncology patients, most commonly in low-grade (94\%) and high-grade gliomas (82\%). Responders reported using preoperative fMRI a median of 10 times (IQR 4-25 times) in the past 12 months. Overall, responders use fMRI in neurooncology patients a median of $24 \%$ (IQR 9\%-41\%) of the time, on low-grade gliomas 38\% (IQR 15\%-54\%) of the time, and on high-grade gliomas $30 \%$ (IQR $10 \%-$ $40 \%$ ) of the time (Table 2).

\section{Decision Factors for Ordering fMRI}

Among the 28 clinical and radiographic indicators, responders scored functional location (median 100, IQR 93-100), aphasia (median 89, IQR 75-100), dominant hemisphere (median 82, IQR 76-100), and motor symptoms (median 84, IQR 73-98) as the most important factors that influence their decision to order an fMRI (on a scale from 0-100). Other important decision factors in- 


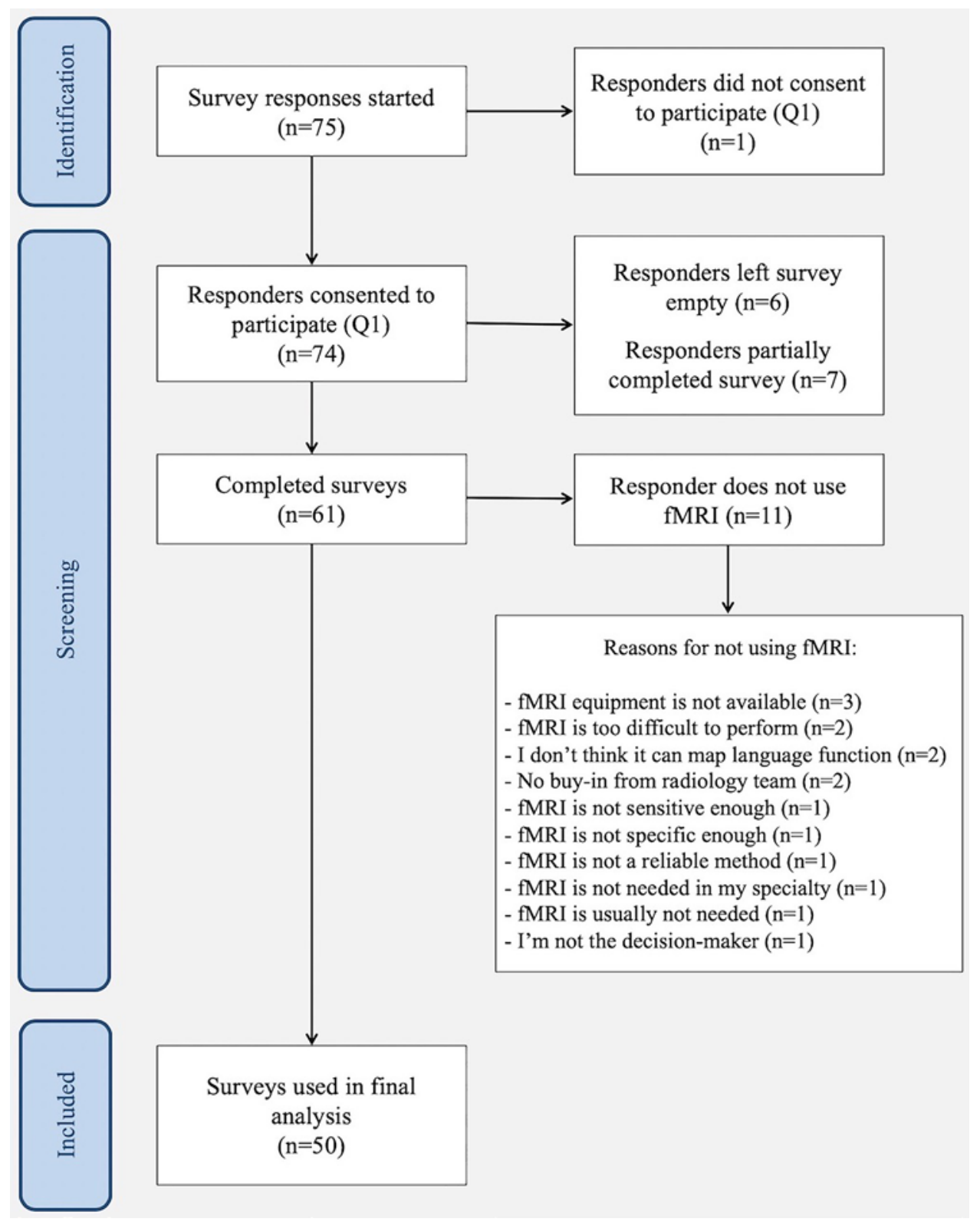

FIG. 1. Flowchart of survey response screening and inclusion. Q1 = question 1.

cluded the following: unilateral tumor with unclear dominance (median 78, IQR 52-86), hemisphere (median 76, IQR 55-90), intention to perform awake surgery (median 65, IQR 46-96), neurological symptoms (median 60, IQR 45-78), sensory symptoms (median 59, IQR 36-78), appearance of infiltration on scan (median 58, IQR 49-79), diffuse versus compact radiographic characteristic (median 55, IQR 20-77), and visual disturbance symptoms (median 53, IQR 2-81; Fig. 2).

\section{Case Studies}

The case studies revealed that the overall agreement between neurosurgeons in ordering fMRI testing was fair (Fleiss' $\kappa=0.300)$. The interrater reliability was highest with regard to the ordering of fMRI for language testing $(\kappa$
$=0.437)$, whereas it yielded fair agreement with regard to the ordering of sensorimotor $(\kappa=0.235)$ and motor fMRI testing (0.256), and slight agreement for the visual domain $(\kappa=0.145)$. Analysis of the case-level interrater reliability revealed 1 case with moderate agreement, 4 with fair agreement, 4 with slight agreement, and 1 with agreement less than chance (Table 3). Case 8 had the least agreement $(\kappa=-0.009)$; this case involved a 62-year-old woman presenting with 2-3 months of gradual personality changes and vision loss, with a large frontal lesion on MRI (see scans in Supplement 1). Case 9 had the most agreement ( $\kappa$ $=0.408$ ); this case involved a 51-year-old man presenting with 1 week of electric-like shocks, left-foot shaking, and left lower-extremity twitching, with a small posterior parietal lesion on MRI (see scans in Supplement 1). 
TABLE 1. Responder demographics and areas of practice

\begin{tabular}{|c|c|}
\hline Survey Question & Value \\
\hline \multicolumn{2}{|l|}{$\begin{array}{l}\text { How many years have you been a practicing neurosur- } \\
\text { geon (i.e., years since finishing residency)? }\end{array}$} \\
\hline None, still in residency & $9(18)$ \\
\hline $0-2$ yrs & $6(12)$ \\
\hline $3-5$ yrs & $7(14)$ \\
\hline $6-9$ yrs & $3(6)$ \\
\hline $10-19$ yrs & $5(10)$ \\
\hline $20+y r s$ & $20(40)$ \\
\hline \multicolumn{2}{|l|}{ What is your gender? } \\
\hline Female & $7(14)$ \\
\hline Male & $43(86)$ \\
\hline \multicolumn{2}{|l|}{ What is your primary patient population? } \\
\hline Adult & $45(90)$ \\
\hline Pediatric & $5(10)$ \\
\hline $\begin{array}{l}\text { If you work with a pediatric population, what do you feel is } \\
\text { the minimum age for which preoperative fMRI can be } \\
\text { used? }\end{array}$ & $\begin{array}{l}\text { Median } 7 \\
\text { yrs (IQR } \\
6-8 \text { yrs) }\end{array}$ \\
\hline \multicolumn{2}{|l|}{ Select your area(s) of medical practice } \\
\hline Academic & $44(88)$ \\
\hline Private & $2(4)$ \\
\hline Both & $3(6)$ \\
\hline Missing & $1(2)$ \\
\hline \multicolumn{2}{|l|}{ Select all areas of your neurosurgical practice } \\
\hline Neurooncology & $50(100)$ \\
\hline Skull base & $31(62)$ \\
\hline Hydrocephalus & $23(46)$ \\
\hline Trauma & $20(40)$ \\
\hline Spine & $17(34)$ \\
\hline Stereotactic and functional (including epilepsy surgery) & $16(32)$ \\
\hline Pediatric neurosurgery & $10(20)$ \\
\hline Cerebrovascular (including endovascular) & $9(18)$ \\
\hline Peripheral nerve & $8(16)$ \\
\hline Neurocritical care & $7(14)$ \\
\hline \multicolumn{2}{|l|}{ What is your main area of specialty in neurosurgery? } \\
\hline Neurooncology & $24(48)$ \\
\hline Skull base & $10(20)$ \\
\hline Pediatric neurosurgery & $6(12)$ \\
\hline Stereotactic and functional (including epilepsy surgery) & $3(6)$ \\
\hline Cerebrovascular (including endovascular) & $2(4)$ \\
\hline Spine & $2(4)$ \\
\hline Peripheral nerve & $0(0)$ \\
\hline Hydrocephalus & $0(0)$ \\
\hline Trauma & $0(0)$ \\
\hline Neurocritical care & $0(0)$ \\
\hline Other/missing & $3(6)$ \\
\hline
\end{tabular}

All data given as number (\%) unless otherwise indicated.
TABLE 2. Preoperative fMRI use by patient population

\begin{tabular}{cc}
\hline \multicolumn{1}{c}{ Survey Question } & Value \\
\hline $\begin{array}{c}\text { On which patients do you use fMRI for surgical } \\
\text { planning? (select all that apply) }\end{array}$ \\
\hline Neurooncology & $50(100)$ \\
\hline Stereotactic and functional neurosurgery & $15(30)$ \\
\hline Cerebrovascular (including endovascular) & $5(10)$ \\
\hline Pediatric neurosurgery & $5(10)$ \\
\hline Skull base & $4(8)$ \\
\hline Peripheral nerve & $0(0)$ \\
\hline Spine & $0(0)$ \\
\hline Trauma & $0(0)$ \\
\hline Hydrocephalus & $50(0)$ \\
\hline $\begin{array}{c}\text { Do you currently use fMRI for the surgical planning } \\
\text { of neurosurgical patients? }\end{array}$ & \\
\hline $\begin{array}{c}\text { Approximately how many times have you used } \\
\text { fMRI for the surgical planning of neurooncology }\end{array}$ & $(\mathrm{IQR} 4-25)$ \\
\hline patients in the past 12 months? & \\
\hline For which neurooncology patients do you use \\
preoperative fMRI? (select all that apply) \\
\hline Neurooncology patients \\
\hline Low-grade glioma
\end{tabular}

All data given as number (\%) unless otherwise indicated.

"Value for those who answered "yes."

$\dagger$ Median percentage of the time (IQR).

\section{Intended Purpose for Ordering fMRI}

The most common goals for ordering fMRI were to: identify language laterality $(n=46,92 \%)$, plan extent of resection $(\mathrm{n}=44,88 \%)$, and discuss neurological risks with the patient $(n=43,86 \%)$. In response to how useful they thought fMRI was for these purposes (scale 0-100), responders rated "identify language laterality" as a median of 79 (IQR 66-94), "planning extent of resection" as 75 (IQR 50-85), and "discussing neurological risks with patients and family" as 78 (IQR 68-97; Fig. 3).

\section{Feasibility of Obtaining fMRI}

Respondents reported that when fMRI was ordered, results were not obtained a median of $10 \%$ (IQR $8 \%-19 \%$ ) 


\section{Clinical Indications}

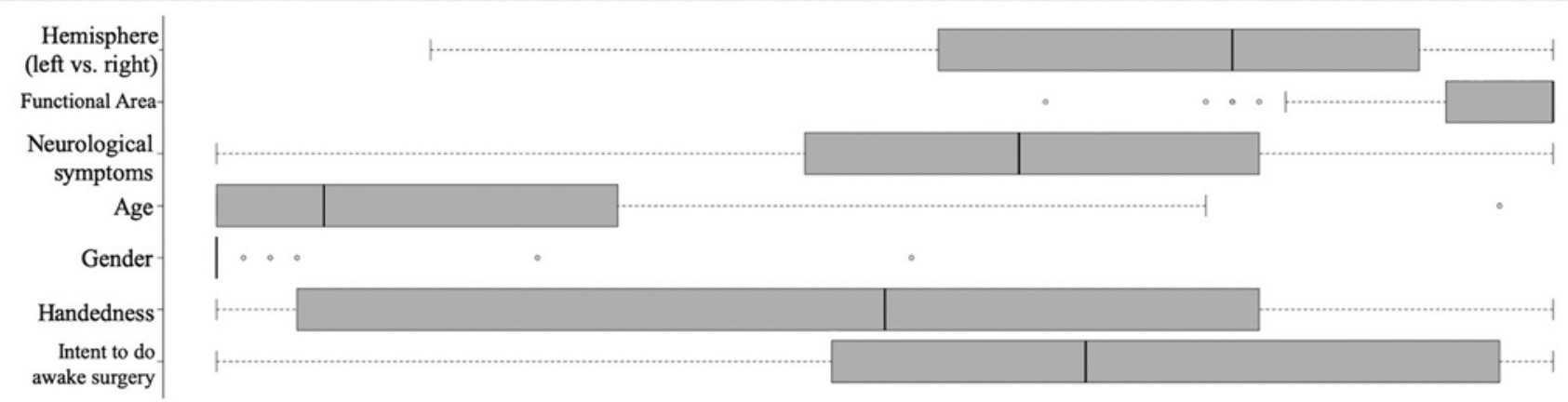

Radiographic Indications

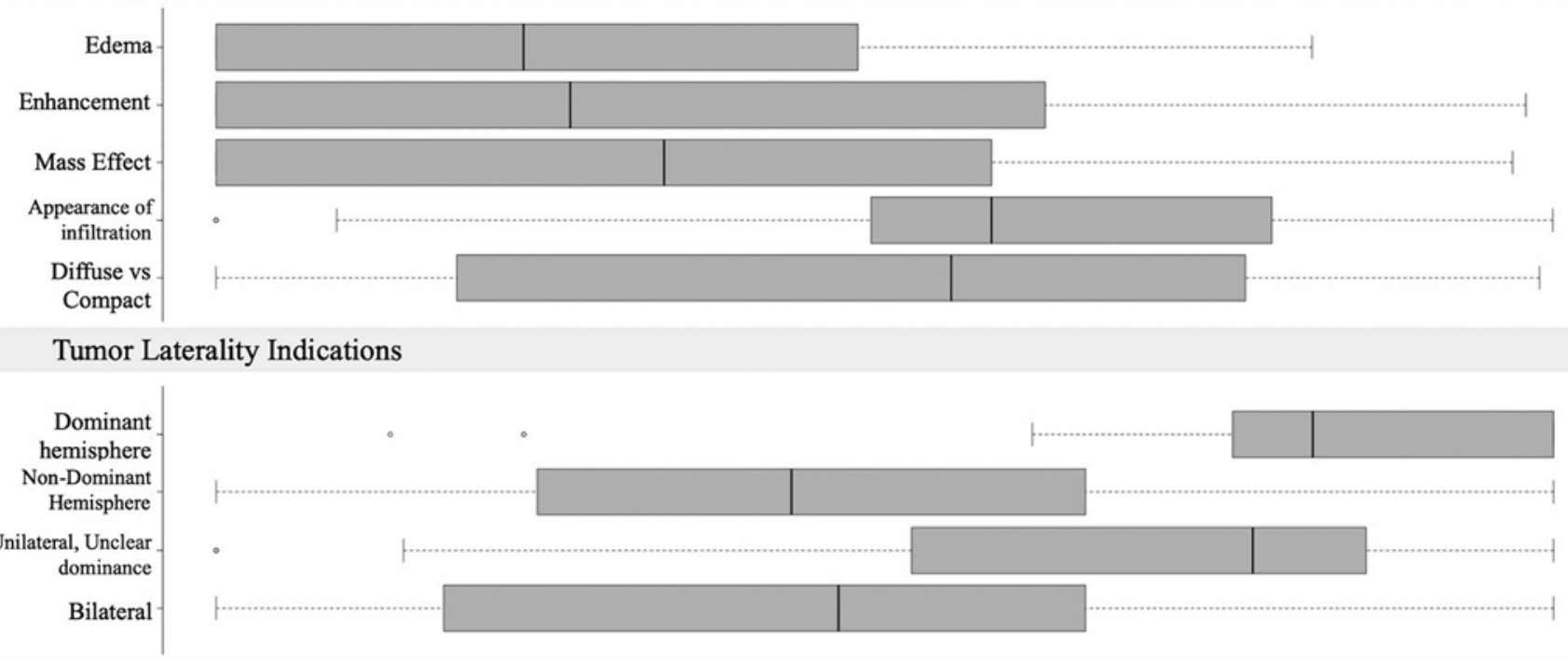

\section{Symptom Indications}

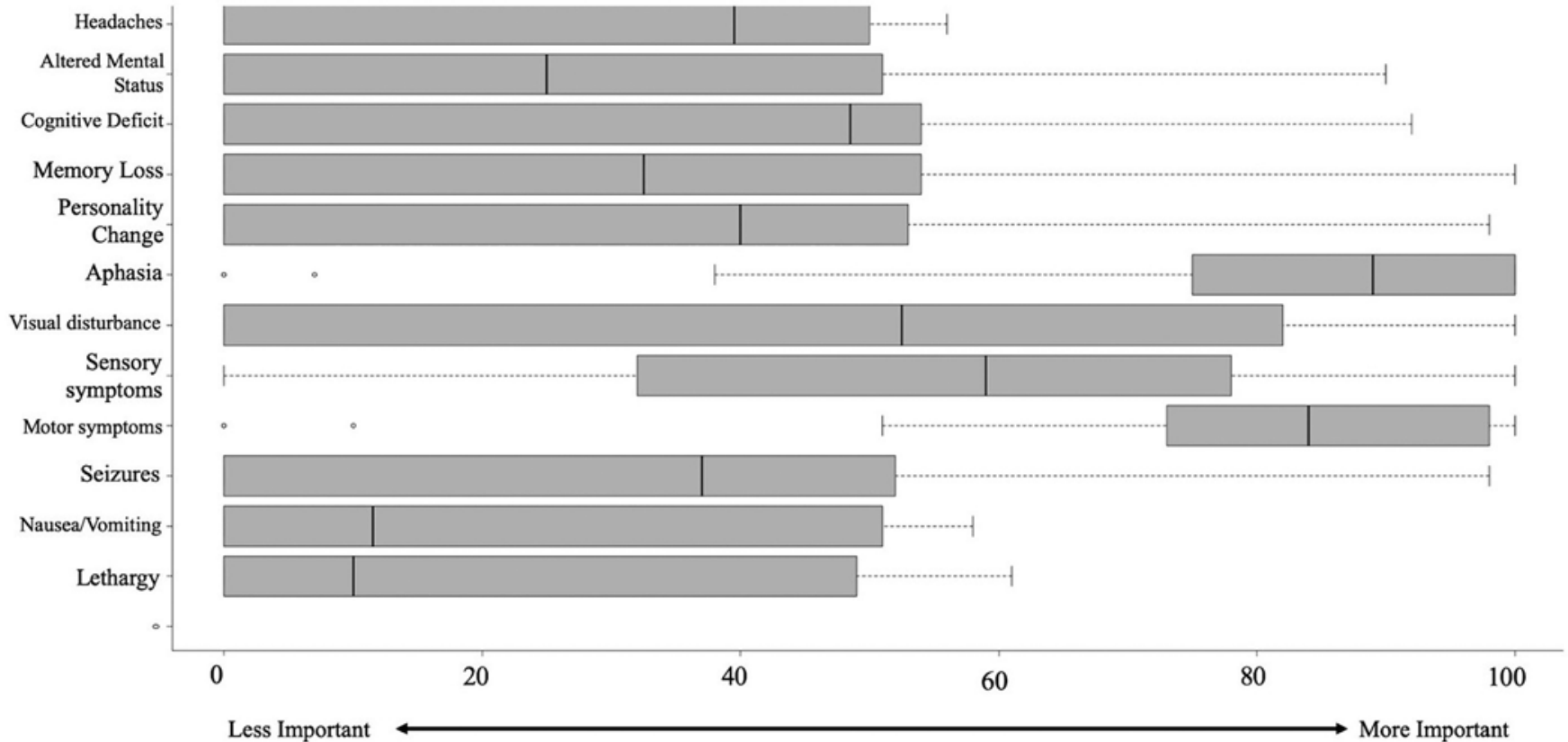

FIG. 2. Boxplot depiction of factors influencing the decision to order fMRI, in response to the question: "Which of the following factors affect your decision to order a preoperative fMRI?" Survey responses were made on a sliding scale of 0-100 (scale from less important to more important). Boxplot represents minimum, first quartile, median, third quartile, and maximum. 
TABLE 3. Interrater reliability among neurosurgeons for ordering preoperative fMRI testing in neurooncology patients

\begin{tabular}{ccl}
\hline \multicolumn{1}{c}{ Analysis Type } & Fleiss' Kappa & \multicolumn{1}{c}{ Interpretation* $^{*}$} \\
\hline Analysis by fMRI testing domain & & \\
\hline Domain & & \\
\hline Overall & 0.300 & Fair agreement \\
\hline Language testing & 0.437 & Moderate agreement \\
\hline Motor testing & 0.256 & Fair agreement \\
\hline Sensorimotor testing & 0.235 & Fair agreement \\
\hline Visual testing & 0.145 & Slight agreement \\
\hline Analysis per case & & \\
\hline Case no. & & \\
\hline 1 & 0.208 & Fair agreement \\
\hline 2 & 0.386 & Fair agreement \\
\hline 3 & 0.300 & Fair agreement \\
\hline 4 & 0.032 & Slight agreement \\
\hline 5 & 0.136 & Slight agreement \\
\hline 6 & 0.168 & Slight agreement \\
\hline 7 & 0.244 & Fair agreement \\
\hline 8 & -0.009 & Agreement less than \\
\hline 9 & & chance \\
\hline 10 & 0.408 & Moderate agreement \\
\hline
\end{tabular}

* The interrater agreement for the consensus labels was calculated using the Fleiss' kappa statistic. The strength of the interrater agreement can be categorized according to this score as less than chance $(<0)$, slight $(0.01-0.20)$, fair $(0.21-0.40)$, moderate $(0.41-0.60)$, substantial $(0.61-0.80)$, and near perfect $(0.81-0.99)$, as given by Viera and Garrett. ${ }^{15}$ of the time and the results were suboptimal a median of $27 \%$ (IQR $16 \%-35 \%$ ) of the time. When queried for why adequate fMRI results were unable to be obtained, most respondents reported that either the patient was unable to adequately perform the task $(\mathrm{n}=38,76 \%)$, the patient was unable to lie still during the scan $(\mathrm{n}=35,70 \%)$, or there was excess head motion during the scan $(n=26,52 \%$; Table 4).

\section{Use of fMRI Results}

Thirty-five responders (70\%) reported that they had at some time resected an fMRI-positive functional site. When queried for why they did so, 27 (77\%) responded that the site was cleared using direct stimulation, 4 (11\%) did not believe anatomically that it was a language area, $7(20 \%)$ that if there was any deficit it would most likely be temporary, and $9(26 \%)$ that the patient was willing to accept a postoperative language deficit. Twenty-nine responders $(58 \%)$ reported that at some time they opted out of resection due to the results of fMRI (Table 5).

\section{Comparison to Other Brain Mapping Techniques}

Of the intraoperative brain mapping options, the greatest portion of respondents is trained in awake surgery (n $=42,84 \%$ ), while sensorimotor mapping is the most regularly used $(\mathrm{n}=31,62 \%)$. Overall, 49 responders $(98 \%)$ reported that if the results of fMRI and intraoperative mapping disagreed, they would rely on the intraoperative mapping. On average, respondents reported that $30 \%$ (IQR $15 \%-43 \%$ ) of the time they found a disagreement between fMRI and awake surgery. While they report that fMRI has

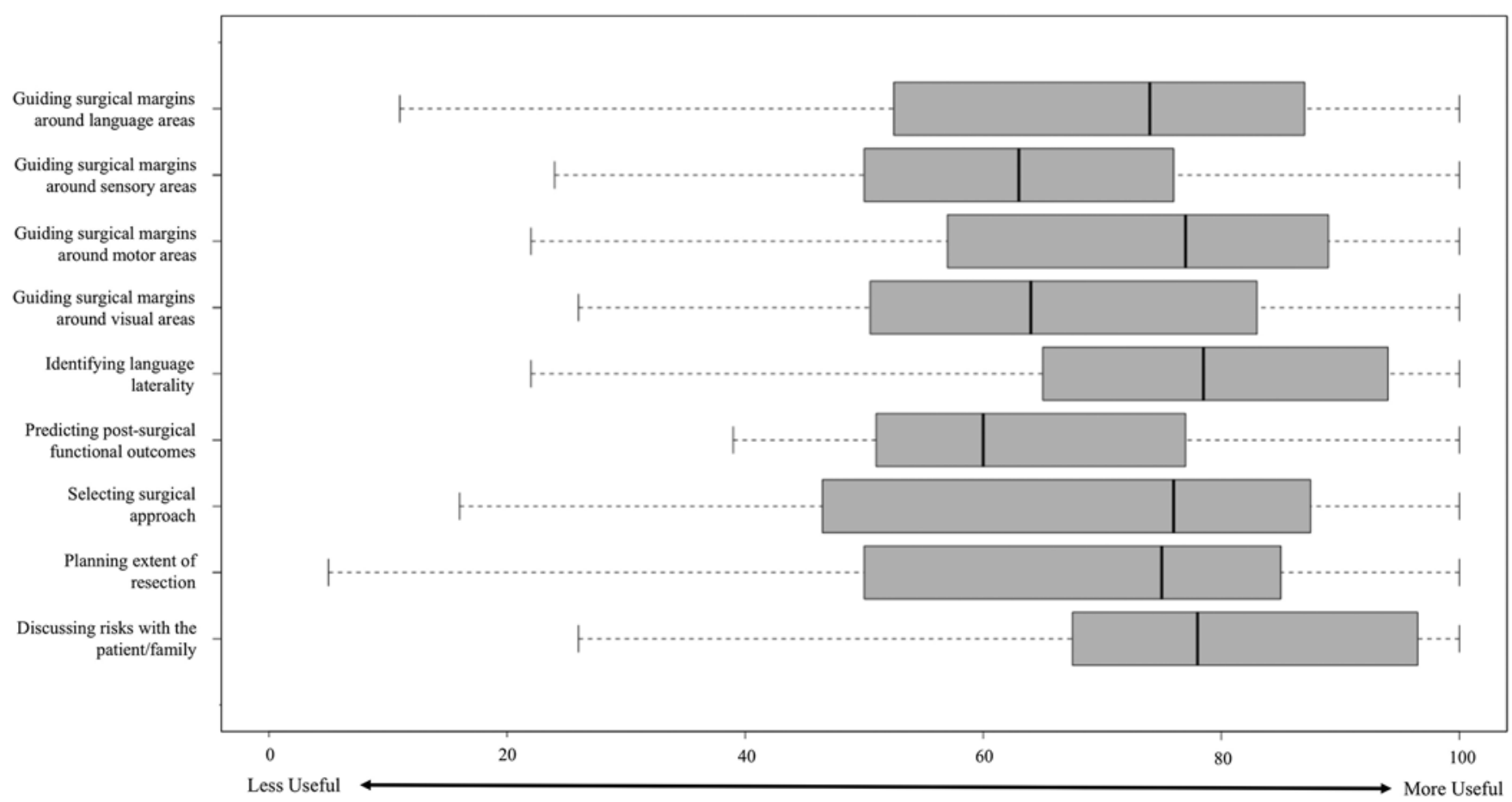

FIG. 3. Boxplot depiction of survey responders' perceived utility of $f M R I$, in response to the question: "How useful do you think preoperative fMRI is for the following purposes?" Survey responses were made on a sliding scale of 0-100 (scale from less useful to more useful). Boxplot represents minimum, first quartile, median, third quartile, and maximum. 
TABLE 4. Feasibility of obtaining fMRI

\begin{tabular}{lc}
\hline \multicolumn{1}{c}{ Survey Question } & Value \\
\hline $\begin{array}{l}\text { When you order a preoperative fMRI, approximately } \\
\text { how often were results not obtained? }\end{array}$ & $\begin{array}{c}\text { Median } 10 \% \\
(I Q R) 8 \%-19 \%)\end{array}$ \\
\hline $\begin{array}{l}\text { When you order a preoperative fMRI, approximately } \\
\text { how often were results suboptimal? }\end{array}$ & $\begin{array}{c}\text { Median } 27 \% \\
(\text { IQR } 16 \%-35 \%)\end{array}$ \\
\hline $\begin{array}{l}\text { For what reasons have you been unable to obtain } \\
\text { an adequate fMRI? (select all that apply) }\end{array}$ & \\
\hline Patient did not understand the instructions & $20(40 \%)$ \\
\hline Patient unable to adequately perform task & $38(76 \%)$ \\
\hline Patient unable to lie still during scan & $35(70 \%)$ \\
\hline Excess head motion during scan & $26(52 \%)$ \\
\hline Patient refused & $6(12 \%)$ \\
\hline Technical problem & $12(24 \%)$ \\
\hline Insurance coverage problem & $10(20 \%)$ \\
\hline Length of time to do testing & $13(26 \%)$ \\
\hline Adverse event & $1(2 \%)$ \\
\hline
\end{tabular}

a strong effect on the decision to perform awake surgery (73\%, IQR 53\%-81\%), fMRI is not a substitute for awake surgery (24\%, IQR 2\%-38\%). Thirty-eight respondents (76\%) use diffusion tensor imaging (DTI), of whom 30 (79\%) use fMRI to guide the tractography and 18 (47\%) use the tractography to guide the interpretation of fMRI results (Table 5).

\section{Discussion}

In surveying neurosurgeons about their perceptions and use of preoperative fMRI for neurooncology patients, we found that there is variability between clinicians. This variability extended across the breadth of the study, including which neurooncology patients should undergo preoperative fMRI, which factors influence the decision to order fMRI, which fMRI to order in the case studies, what purposes fMRI is used for, and the utility of fMRI relative to other brain mapping techniques. These results reflect different experiences with and attitudes toward fMRI, all of which are useful for informing the future of this imaging modality as a clinical tool.

TABLE 5. Comparative performance of fMRI

\begin{tabular}{|c|c|}
\hline Survey Question & Value \\
\hline \multicolumn{2}{|l|}{ If you have ever resected fMRI-identified, functional positive sites, why did you do so? (select all that apply) } \\
\hline I have never done this & $15(30)$ \\
\hline Total have done so & $35(70)$ \\
\hline The area was cleared using direct stimulation & $27(77)$ \\
\hline We did not believe anatomically that it was a language area & $4(11)$ \\
\hline If there was any deficit, it would most likely be temporary & $7(20)$ \\
\hline Patient was willing to accept a postsurgical language deficit & $9(26)$ \\
\hline Have you ever opted not to pursue surgical resection due to the results of preoperative fMRI? & $29(58)$ \\
\hline \multicolumn{2}{|l|}{ If you use intraoperative brain mapping, please select all the techniques that you use (select all that apply) } \\
\hline I am trained in awake surgery & $42(84)$ \\
\hline I regularly perform awake surgery & $22(44)$ \\
\hline I am trained in language mapping & $36(72)$ \\
\hline I regularly perform language mapping & $21(42)$ \\
\hline I am trained in sensorimotor mapping & $41(82)$ \\
\hline I regularly perform sensorimotor mapping & $31(62)$ \\
\hline \multicolumn{2}{|l|}{ When there is disagreement between the results of intraoperative mapping and fMRI, which do you rely on more? } \\
\hline Rely more on intraoperative mapping & $49(98)$ \\
\hline Rely more on fMRI & $1(2)$ \\
\hline $\begin{array}{l}\text { In patients who underwent both fMRI and awake surgery, approximately how often was there a disagreement } \\
\text { between fMRI and awake surgery? }\end{array}$ & Median 30\% (IQR 15\%-43\%) \\
\hline \multicolumn{2}{|l|}{ In your experience, preoperative fMRI...* } \\
\hline ...results have a strong effect on your decision to do awake surgery & Median 73\% (IQR 53\%-81\%) \\
\hline ...serves as an indication to perform awake surgery & Median $75 \%$ (IQR 55\%-81\%) \\
\hline ...can substitute for awake surgery & Median 24\% (IQR 2\%-38\%) \\
\hline \multicolumn{2}{|l|}{ If you use preoperative fMRI with DTI (diffuse tensor imaging), please select all that apply } \\
\hline I use fMRI to see the tractography & $30(79)$ \\
\hline I use the tractography to guide the fMRI & $18(47)$ \\
\hline I do not use DTI & $12(24)$ \\
\hline
\end{tabular}

All data given as number (\%) unless otherwise indicated.

* Scale $0-100(0=$ strongly disagree, $100=$ strongly agree $)$. 
Among the survey responders who reported not using fMRI, their reasons included that fMRI is too difficult to perform, that it is not sensitive or specific enough, and that it is not believed to be able to map language function. While this is the first study to quantify neurosurgeons' perspectives on preoperative fMRI for neurooncology patients, this finding is reflected qualitatively in the literature. ${ }^{8,14}$ Given the advances in technology and research that reflect a strong case for clinical use of fMRI, ${ }^{11,16}$ there may be an opportunity to disseminate updated reviews and guidelines.

Among those who do currently use fMRI for neurooncology patients, we found the median use is 10 times in the past 12 months, and it is used on 24\% (IQR 9\%-41\%) of neurooncology patients. So while its use has increased over time, fMRI is still not used in the preoperative planning of most patients. As Rosen and Savoy explored at the 20-year anniversary of fMRI's inception, fMRI has clearly made an impact scientifically, but its impact medically is not yet "world-changing." 2 In comparison to the ubiquitous use of other imaging modalities, fMRI still has some room to grow in its clinical adoption, such as the use of resting state that may allow more patients to be mapped with fMRI.

The variability of clinical and radiographic indications that influence the decision to order preoperative fMRI is illustrated in Fig. 2. While there are few factors on which clinicians strongly agree, one is that functional area is a clinical indication for fMRI. Others, such as memory loss and handedness, present with such variability that the answers ranged across the entire 0-100 scale of importance. Furthermore, the disagreement across fMRI testing domains and case studies, as illustrated in Table 3 , reveals some important differences in opinion regarding the clinical application of fMRI. The diverse opinions come from neurosurgeons around the country, practicing in various different settings, and so these findings may be able to open the conversation to what clinical factors should signal the need for fMRI. The discrepancies should be taken into consideration to develop guidelines for best practices surrounding preoperative fMRI testing in neurooncology patients.

Responders differed in their perspectives on what purpose fMRI can serve, but identification of language laterality was the most agreed-upon purpose (92\%), and in the case studies, language fMRI testing yielded the highest interrater reliability $(\kappa=0.437)$. These findings concur with the survey study from Benjamin et al., which found that fMRI was most commonly used to identify the dominant language hemisphere in the preoperative planning of epilepsy patients. ${ }^{3}$ Furthermore, research into this use of fMRI has yielded compelling evidence for the reliable use of fMRI in assessing language laterality. ${ }^{2}$ In fact, language assessment is the only presurgical fMRI guideline published to date by the American Society of Functional Neuroradiology (ASFNR). ${ }^{4}$ The ASFNR 2017 guidelines for presurgical language assessment by means of fMRI provided the first step toward standardizing fMRI data across institutions, and following this success there may be the opportunity to explore other aspects of presurgical fMRI use that are presently subject to interclinician variability.
Interestingly, $70 \%$ of responders report having resected an fMRI-positive site at one time or another. Yet, 58\% of responders have ever opted out of resection due to fMRI results. On the one hand, these seemingly contradictory results could reflect the significant variation in use and presumed utility across different practices. On the other hand, this dichotomy could reflect both the confidence in fMRI and understanding of its potential shortcomings. Examining this dichotomy could be instrumental for combining fMRI with other modalities for functional mapping and developing guidelines and best practices on a national scale.

Furthermore, $98 \%$ of responders said they would rely on the intraoperative mapping results if the results of fMRI and intraoperative mapping disagreed, which indicates that intraoperative mapping remains the perceived gold standard for preoperative neurosurgical planning. Because responders report that there is disagreement between fMRI and awake surgery $30 \%$ of the time, further research is needed to understand the nature of these discrepancies. From this clinician survey, we have identified several areas in which further research is needed. Future studies may include patient outcome studies, and quantitative studies correlating fMRI findings with intraoperative mapping findings.

\section{Strengths and Limitations}

A few limitations should be mentioned. The majority of responses (88\%) came from neurosurgeons working in academic centers. As such, this survey provides limited insight into the use of fMRI for brain tumor resection in nonacademic centers. Although the survey was distributed across various neurosurgical departments in the US, participation remains self-selective. This could have resulted in a nonrepresentative group of responders, which could be biased toward users and those in favor of fMRI. Yet, this survey does provide valuable information on the nonuser group as well. Despite the limitations, we believe this study provides valuable insight into the current use and presumed utility of preoperative fMRI for brain tumor resection among neurosurgeons in the US.

\section{Conclusions}

In this survey among neurosurgeons, we found a substantial degree of variability in the current use and presumed utility of fMRI for preoperative surgical planning in brain tumor patients. This variation encompasses clinical and radiographic indications for ordering fMRI, functional domains to test in specific case studies, and clinical purposes of using fMRI. In recent years, clinical use of fMRI has increased, and clinicians have gained valuable and varied experiences with fMRI, which can now be leveraged for the collective development of best practices. A crucial next step toward increasing the clinical adoption of fMRI will be to develop a comprehensive set of guidelines that inform where and when to implement fMRI in the preoperative planning of neurooncology surgical patients.

\section{Acknowledgments}

We would like to thank all of the clinicians who voluntarily 
agreed to participate in our survey and took the time to contribute to the study. We would also like to thank Courtney Gilligan, whose help was instrumental in compiling the email distribution list used for this study.

\section{References}

1. Bandettini PA: Twenty years of functional MRI: the science and the stories. Neuroimage 62:575-588, 2012

2. Bauer PR, Reitsma JB, Houweling BM, Ferrier CH, Ramsey NF: Can fMRI safely replace the Wada test for preoperative assessment of language lateralisation? A meta-analysis and systematic review. J Neurol Neurosurg Psychiatry 85:581588, 2014

3. Benjamin CFA, Li AX, Blumenfeld H, Constable RT, Alkawadri R, Bickel S, et al: Presurgical language fMRI: clinical practices and patient outcomes in epilepsy surgical planning. Hum Brain Mapp 39:2777-2785, 2018

4. Black DF, Vachha B, Mian A, Faro SH, Maheshwari M, Sair HI, et al: American Society of Functional Neuroradiologyrecommended fMRI paradigm algorithms for presurgical language assessment. AJNR Am J Neuroradiol 38:E65E73, 2017

5. Buchbinder BR: Functional magnetic resonance imaging. Handb Clin Neurol 135:61-92, 2016

6. Dimou S, Battisti RA, Hermens DF, Lagopoulos J: A systematic review of functional magnetic resonance imaging and diffusion tensor imaging modalities used in presurgical planning of brain tumour resection. Neurosurg Rev 36:205-214, 2013

7. Goodman LA: Snowball sampling. Ann Math Stat 32:148170,1961

8. Haller S, Bartsch AJ: Pitfalls in FMRI. Eur Radiol 19:26892706, 2009

9. Logothetis NK: What we can do and what we cannot do with fMRI. Nature 453:869-878, 2008

10. McHugh ML: Interrater reliability: the kappa statistic. Biochem Med (Zagreb) 22:276-282, 2012

11. Orringer DA, Vago DR, Golby AJ: Clinical applications and future directions of functional MRI. Semin Neurol 32:466475, 2012

12. Rosen BR, Savoy RL: fMRI at 20: has it changed the world? Neuroimage 62:1316-1324, 2012
13. Silva MA, See AP, Essayed WI, Golby AJ, Tie Y: Challenges and techniques for presurgical brain mapping with functional MRI. Neuroimage Clin 17:794-803, 2017

14. Sunaert S: Presurgical planning for tumor resectioning. J Magn Reson Imaging 23:887-905, 2006

15. Viera AJ, Garrett JM: Understanding interobserver agreement: the kappa statistic. Fam Med 37:360-363, 2005

16. Vysotski S, Madura C, Swan B, Holdsworth R, Lin Y, Rio AMD, et al: Preoperative fMRI associated with decreased mortality and morbidity in brain tumor patients. Interdiscip Neurosurg 13:40-45, 2018

\section{Disclosures}

The authors report no conflict of interest concerning the materials or methods used in this study or the findings specified in this paper.

\section{Author Contributions}

Conception and design: Golby, Senders. Acquisition of data: Stopa. Analysis and interpretation of data: Stopa, Senders. Drafting the article: Stopa, Senders. Critically revising the article: Golby, Broekman, Vangel. Reviewed submitted version of manuscript: all authors. Approved the final version of the manuscript on behalf of all authors: Golby. Statistical analysis: Stopa, Senders, Vangel. Study supervision: Golby, Broekman.

\section{Supplemental Information}

Online-Only Content

Supplemental material is available online.

Supplement 1. https://thejns.org/doi/suppl/10.3171/2019.11. FOCUS19779.

\section{Correspondence}

Alexandra J. Golby: Brigham and Women's Hospital, Harvard Medical School, Boston, MA. agolby@bwh.harvard.edu. 